INPLASY

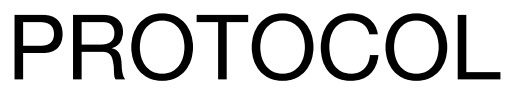

To cite: Xu et al. Efficacy of estazolam on daytime symptoms in patients with insomnia - A systematic review and meta-analysis. Inplasy protocol 2021110040. doi:

10.37766/inplasy2021.11.0040

Received: 12 November 2021

Published: 12 November 2021

Corresponding author:

Lei $\mathrm{Xu}$

prosperityzy@163.com

Author Affiliation:

Beijing university of Chinese medicine.

Support: No. 2018YFC1705700.

Review Stage at time of this submission: Data analysis.

Conflicts of interest:

None declared.

\section{Efficacy of estazolam on daytime symptoms in patients with insomnia - A systematic review and meta-analysis}

Xu, L1; Wang, X²; Zhang, L3; Yao, Z4; Qiang, R5; Lv, W6.

Review question / Objective: P:Patients with insomnia diagnosed by Snternational Classification of Sleep Disorders (ICSD), Diagnostic and Statistical Manual of Mental Disorders (DSM), Vnternational Classification of Diseases (CD), regardless of age, sex. I:Inclusion in the study should include at least one group for estazolam treatment of insomnia.But the combination of drugs is ruled out. C:Control group is placebo. But the combination of drugs is ruled out. O:Outcomes:1.) daytime function, 2.) daytime sleepiness, 3.) anxiety, 4.) depression, 5.) cognitive function, 6.) adverse reactions, 7.) compliance.

Information sources: Cochrane Central Register of Controlled Trials (CENTRAL), PubMed, EMBASE. China National Knowledge Network (CNKI), Wanfang, VIP and CBM Sinomed.

INPLASY registration number: This protocol was registered with the International Platform of Registered Systematic Review and Meta-Analysis Protocols (INPLASY) on 12 November 2021 and was last updated on 12 November 2021 (registration number INPLASY2021110040).

\section{INTRODUCTION}

Review question / Objective: P:Patients with insomnia diagnosed by Snternational Classification of Sleep Disorders (ICSD), Diagnostic and Statistical Manual of Mental Disorders (DSM), Vnternational Classification of Diseases (CD), regardless of age, sex. I:Inclusion in the study should include at least one group for estazolam treatment of insomnia. But the combination of drugs is ruled out. C:Control group is placebo. But the combination of drugs is ruled out. 0:Outcomes:1.) daytime function, 2.) daytime sleepiness, 3.) anxiety, 4.) depression, 5.) cognitive function, 6.) adverse reactions, 7 .) compliance. 
Condition being studied: The current incidence rate of the world's population is about 10 per cent, which places a serious burden on society. Insomniacs reported higher rates of absenteeism, as well as higher overall medical costs.Up to $40 \%$ of people with insomnia have a common mental illness, the most common being generalized anxiety disorder and major depression.Perhaps most striking, however, are similar findings from at least six studies showing that people with insomnia who currently do not have mood disorders have a significantly increased risk of developing major depression more than a decade later. Weissman reported that the odds ratio for insomniacs who subsequently progressed to major depression was 5.4. There is also data showing that the greater the variety of sleep symptoms, the greater the risk of subsequent depression.

\section{METHODS}

Participant or population: This review will include patients with insomnia diagnosed by Snternational Classification of Sleep Disorders (ICSD), Diagnostic and Statistical Manual of Mental Disorders (DSM), Vnternational Classification of Diseases (CD), regardless of age, sex.

Intervention: Inclusion in the study should include at least one group for estazolam treatment of insomnia.But the combination of drugs is ruled out.

Comparator: Control group is placebo. But the combination of drugs is ruled out.

Study designs to be included: RCTs assessing efficacy of estazolam on daytime symptoms in patients with insomnia will be included.Reviews, systematic reviews, case reports, editorials and study protocols will be excluded.

Eligibility criteria: Included if the following conditions are met:(1)Research type:RCTs; (2)Patients with insomnia diagnosed by Snternational Classification of Sleep Disorders (ICSD), Diagnostic and Statistical Manual of Mental Disorders (DSM),
Vnternational Classification of Diseases (CD), regardless of age, sex.(3)Inclusion in the study should include at least one group for estazolam treatment of insomnia.But the combination of drugs is ruled out. (4)Control group is placebo. But the combination of drugs is ruled out.

Information sources: Cochrane Central Register of Controlled Trials (CENTRAL), PubMed, EMBASE. China National Knowledge Network (CNKI), Wanfang, VIP and CBM Sinomed.

Main outcome(s): 1.) daytime function, 2.) daytime sleepiness, 3.) anxiety, 4.) depression, 5.) cognitive function, 6.) adverse reactions, 7.) compliance.

Additional outcome(s): PSQI.

Quality assessment / Risk of bias analysis: This will assess the categories of bias for each study: selection bias (random sequence generation and allocation concealment), detection bias (blinding of outcome assessment), attrition bias (incomplete outcome data), reporting bias (selective reporting) and other bias (unbalanced base line, early termination). The included literature will be evaluated item by item according to the Risk of Bias 2 tool in the Cochrane Systematic Reviews Manual (Version 5.1.0). The evaluation contents includes 6 items: random sequence generation method, random allocation concealment, blind method, data integrity, selective reporting results, and other bias. The evaluation results will be described as high risk, low risk and unclear, respectively. The included studies will be evaluated by two researchers, and any differences could be resolved through discussion. If no agreement is reached through discussion and consultation, the decision shall be made by a third researcher in the relevant field.

Strategy of data synthesis: Data will be summarized by using risk ratios with $95 \%$ confidence interval (Cl) for dichotomous outcomes or mean difference with $95 \% \mathrm{Cl}$ for continuous outcomes. It is anticipated that different scales may be used to report 
the same outcomes, in which case we will use the standardised mean difference (SMD). Statistical heterogeneity will be assessed using the $I^{2}$ statistic (on the bases of characteristics of the included studies and the participants, details of the intervention or control, and types of outcome measurements). If the $I^{2}$ statistic is $<50 \%$ and the clinical heterogeneity among trials is acceptable, data will be pooled for statistical analyses using the Cochrane' Review Manager software (V.5.3). Fixed effects model will be used to conduct the meta-analysis when the $\mathbf{I}^{2}$ statistic is $50 \%$ ), subgroup analysis or descriptive analysis will be conducted.

Subgroup analysis: Where data are available, subgroup analyses will be conducted to determine if effectiveness of estazolam is influenced by: different kind of treatments.

Sensitivity analysis: The studies were excluded one by one, and then the metaanalysis was performed in the remaining studies.

Language: English.

Country(ies) involved: China.

Keywords: Insomnia, Estazolam, Daytime function, systematic review.

Contributions of each author:

Author 1 - Lei Xu.

Author 2 - Xuejiao Wang.

Author 3 - Lili Zhang.

Author 4 - Zi-ang Yao.

Author 5 - Rui Qiang.

Author 6 - Wenliang Lv. 